\title{
Opportunities for detailed fission studies using light charged particle reac- tions
}

\author{
Birger B. Back ${ }^{1, *}$ \\ ${ }^{1}$ Argonne National Laboratory, 9700 S. Cass Avenue, Lemont, IL 60439, USA
}

\begin{abstract}
Since its discovery in 1939, the nuclear fission process has provided much insight into the behavior of nuclei under many different conditions. As part of the nuclear chain reaction, the fission process has had a profound impact on modern society and it has consequently attracted much attention to the field of nuclear physics. In this talk, I will argue that the time is ripe for a resumption of studies of the fission process induced by light charged particle reactions. Although fission can be induced in heavy nuclei by several means, in some cases these methods suffer from the complication that fission can occur at several points during the decay chain thus mixing up contributions from different excitation energies. Using instead light charged particle reactions to excite the nuclei in question, the precise excitation energy from which fission takes place, can be determined. In fact, a number of such studies were carried out previously, and a first set of results on fission barrier heights, mass, energy and angular distributions were obtained. Applying detection techniques developed over the last decades will allow researchers to obtain detailed, high-quality data from which to probe and refine our present understanding of the process. Based on these observations, I suggest that substantial advances in the study of this process can be achieved by using simple light charged particle reactions.
\end{abstract}

\section{Introduction}

Over the past 80 years, since the discovery of fission [1] and the almost prompt explanation of many aspects of this disruptive nuclear decay channel [2], this phenomenon has been a central testing ground for theories and models aiming to describe both the dynamical and static properties of nuclei. Early on, much insight was gained by using the liquid drop model, which approximates the average properties with those of a charged liquid drop. However, some pertinent properties of fission could not be described in this approach, such as the asymmetric mass distributions observed in fission of actinide nuclei and the extra binding of nuclei at or near magic numbers. In the mid 1960s it was thus realized that methods for incorporating the socalled "shell-effects" were needed. An early attempt to include shell effects on nuclear masses was carried out by Myers and Swiatecki [3] but the effects on fission mass distributions and the fission barrier were not considered in this work. In the following sections, these effects, and how they manifest themselves in experimental observables will be discussed.

\section{Shell effects and the double humped barrier}

The development of the understanding of shell effects on nuclear fission properties was done in close collaboration between theorists and experimentalists trying to understand several puzzling experimental data. First among

*e-mail: back@anl.gov these was the discovery of a short-lived fission activity produced by ${ }^{22} \mathrm{Ne}$ bombardment of a ${ }^{242} \mathrm{Pu}$ target carried out by Polikanov et al. [4] in an attempt to produce element $Z=104$ (now known as Rutherfordium, Rf) in a complete fusion reaction. This activity was, however, much shorter than expected and when the same activity was produced also in the ${ }^{22} \mathrm{Ne}+{ }^{238} \mathrm{U}$ reaction, it was clear that the activity did not belong to Rutherfordium. This activity was finally assigned to an isomer of ${ }^{242} \mathrm{Am}$, the first fission isomer [5]. The final interpretation of this fission isomer, as well as a number of other experimental data, was given by Bjørnholm and Strutinsky [6]. They considered the effects of nuclear shells on the deformation potential of heavy nuclei using the theoretical method developed by Strutinski [7]. The effect on the deformation potential and some of the experimental consequences are illustrated in Fig. 1] When added to the liquid drop barrier shown in the upper part of Fig. [1] a negative shell energy leads a deformed ground state and a second well in the deformation potential. This second well gives rise to the observed short-lived fission isomer since the outer barrier is much easier to penetrate than the full barrier associated with spontaneous fission from the ground state.

\subsection{First clue that was missed}

It is interesting to note that an earlier clue to the existence of a double-humped fission barrier was available in the literature since 1959. In a study of the fission probability of several actinide nuclei, Northrop, Stokes, and Boyer [8] had seen a pronounced bump at $\sim 5 \mathrm{MeV}$ excitation in 


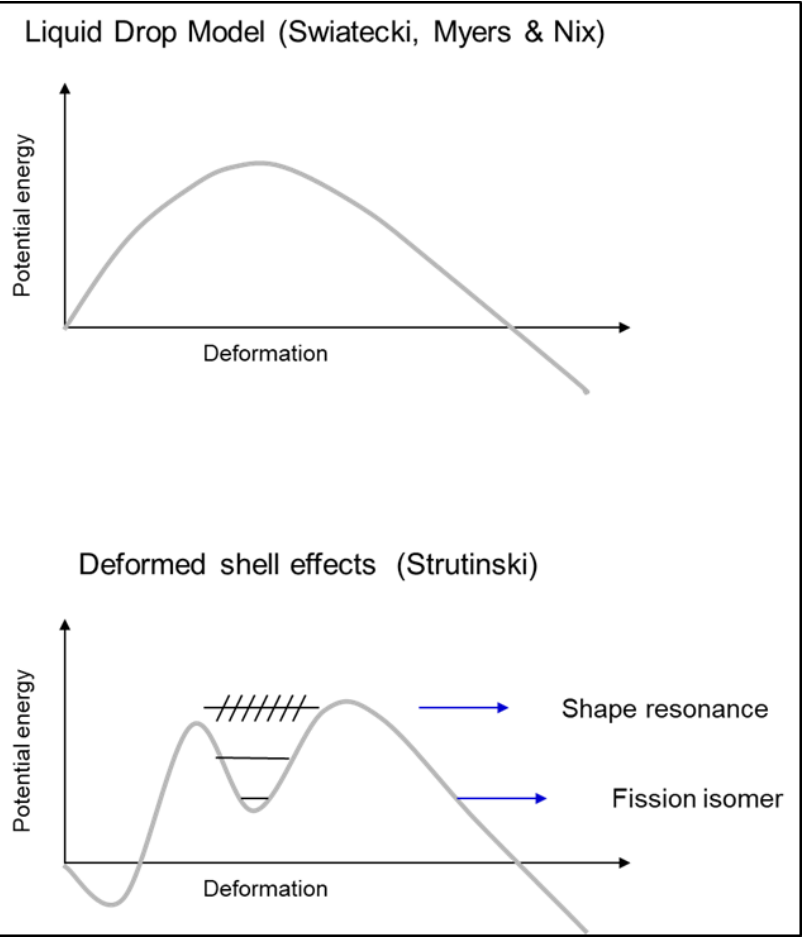

Figure 1. Schematic illustration of how the shell correction to the fission barrier predicted by the Liquid Drop Model (top panel) leads to the double-humped fission barrier (bottom panel), which supports a fission isomer state (the ground state in the second well) and excited $\beta$-vibrational states that manifest themselves as resonances in the fission probability.

${ }^{240} \mathrm{Pu}$ formed in a ${ }^{239} \mathrm{Pu}(\mathrm{d}, \mathrm{pf})$ reaction, see Fig. 2. An attempt was made to explain this bump - or plateau - in terms of two, widely spaced transition states at the fission barrier. However, in retrospect, we now understand this as a vibrational resonance caused by the existence of the second well. This causes the transmission coefficient for compound states in the first well to be enhanced at energies corresponding to the location of $\beta$-vibrational states in the second well as illustrated in Fig. 11, bottom part.

\subsection{Gross structure in neutron resonances}

A second manifestation of the second well in the fission barrier was found in the gross structure of neutron resonances in e.g. neutron capture on ${ }^{240} \mathrm{Pu}$ at low energies $\mathrm{E}_{n}<3 \mathrm{keV}$ as illustrated in Fig. 3 . We observe that the total cross section for neutron capture exhibits a large number of resonances with an average spacing $\mathrm{D}_{\mathrm{I}} \sim 14 \mathrm{eV}$ reflecting the spacing of $\mathrm{I}^{\pi}=1 / 2^{+}$levels in the first well, whereas the fission cross section shows an additional gross structure with a spacing of roughly $\mathrm{D}_{\text {II }} \sim 630 \mathrm{eV}$. This enhancement in the fission decay occurs by coupling to $\mathrm{I}^{\pi}=1 / 2^{+}$states in the second well, which have a larger fission width since only the outer barrier needs to be overcome on the way to scission. Fig. 4 illustrates the separation of Class I and Class II states caused by the inner barrier. This classification scheme refers to the spatial part of the wave function for these compound states. The dif-

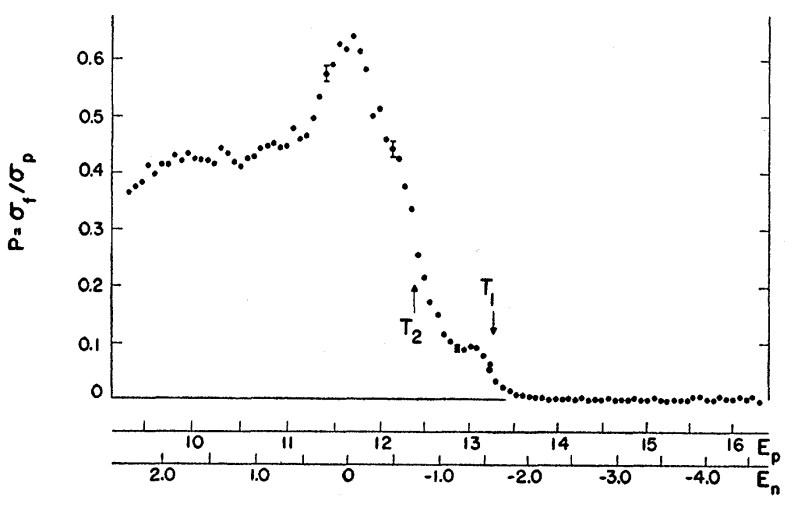

Figure 2. The fission probability measured in ${ }^{239} \mathrm{Pu}(\mathrm{d}, \mathrm{pf})$ reaction at $\mathrm{E}_{d}=14 \mathrm{MeV}$ plotted as a function of the energy, $\mathrm{E}_{p}$, of the outgoing proton taken from Ref. [8]. Note the resonance structure at $\mathrm{E}_{p}=13 \mathrm{MeV}$, which corresponds to an excitation energy of $\sim 5 \mathrm{MeV}$ in the ${ }^{240} \mathrm{Pu}$ nucleus. Note that negative neutron energies (not physical) correspond to excitation energies below the neutron binding energy.

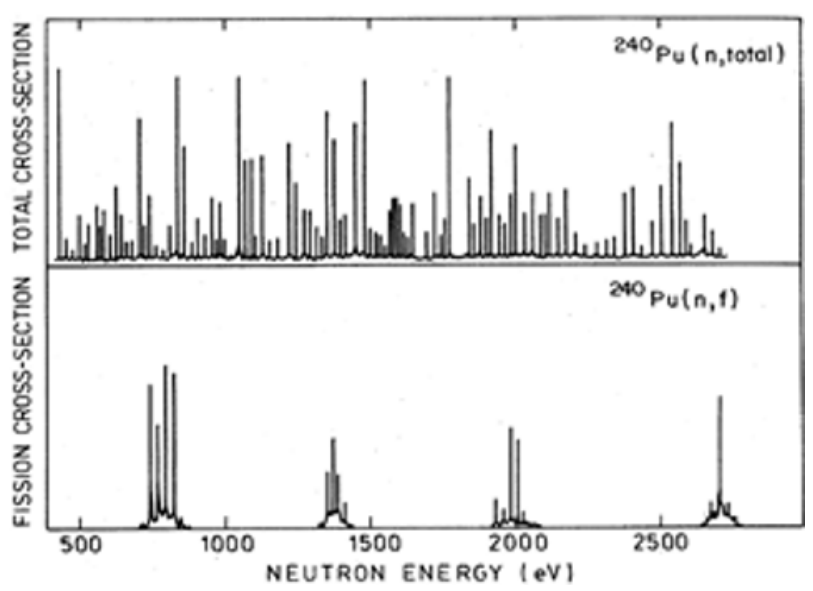

Figure 3. The total ${ }^{240} \mathrm{Pu}$ (n, total) neutron capture cross section measured by Kolar and Böckhoff [9] (upper panel) and the ${ }^{240} \mathrm{Pu}(\mathrm{n}, \mathrm{f})$ cross section measured by Migneco and Theobald [10] (lower panel) are plotted as a function of neutron energy. Note that the fission cross section is strongly enhanced for resonances near Class II states in the second well. The figure is taken from Ref. [11].

ference in level spacing arises from the difference in excitation energy in the two wells.

\subsection{Fission Isomers}

Following the first discovery of fission isomers and the understanding that this may be a general phenomenon based on the Strutinski theory [7], a large effort was undertaken in order to map out the occurrence of this phenomenon. The second well in the deformation potential occurs at a deformation of about $\beta=0.6$. The fission isomers thus represent the first 'super-deformed' states, which were later found to occur also in the $\mathrm{A}=150$ [12] and $\mathrm{A}=190$ [13] regions using gamma spectrometric methods. A map of the island of fission isomers in the actinide region has 


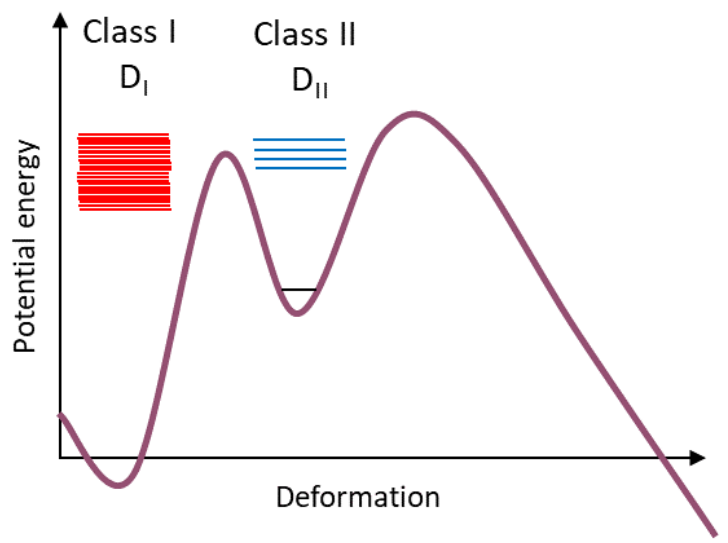

Figure 4. Classification of compound nuclear states into Class I states with average spacing of $D_{I}$ with the main amplitude in the first well and the class II states and average level spacing $\mathrm{D}_{\text {II }}$ belonging to the second well.

been compiled by Bjørnholm and Lynn [11]. Studies of the excitation spectrum in the second well are very rare, but one example is the observation of the rotational states built upon the fission isomer in ${ }^{240} \mathrm{Pu}$ by Specht et al. [14].

\subsection{Vibrational resonances}

While the transmission coefficient through a smooth fission barrier increases monotonously with excitation energy, the existence of the second well in the fission barrier gives rise to resonances at certain excitation energies corresponding to the location of $\beta$-vibrational states in the second well as illustrated in Fig. 5. Although these $\beta$ resonances may be somewhat broadened because of coupling to the underlying compound (Class II) states, in many even-even nuclei they appear as proper resonances in the fission probability function. One example is again ${ }^{240} \mathrm{Pu}$ populated via the ${ }^{239} \mathrm{Pu}(\mathrm{d}, \mathrm{pf})$ reaction [15] in which, with enhanced energy resolution, the fission probability function exhibits a clear resonance at $\mathrm{E}_{X} \sim 5.0 \mathrm{MeV}$, the region where previously only a step was observed [8], see Fig. 6 Subsequent studies of this system have been carried out using a magnetic spectrometer to register the energy of the outgoing proton [16-18]. A substantially better excitation energy resolution $\Delta \mathrm{E}_{X} \sim 5 \mathrm{keV}$, can be achieved using this technique. With this resolution, many details concerning the transition state spectrum at the top of the fission barriers have been revealed. Unfortunately, only a few cases have been studied using this superior technique. An interesting and dramatic $\sim 1 \mathrm{MeV}$ shift in the fission threshold occurs as one crosses the $\mathrm{N}=152$ shell from ${ }^{248} \mathrm{Cm}$ to ${ }^{250} \mathrm{Cm}$ as shown in Fig. 7. The deformed shell and the underlying liquid drop energy that determines the binding at the fission barrier is not expected to change much when crossing the $\mathrm{N}=152$ shell. However, the decreased binding of the ${ }^{250} \mathrm{Cm}$ ground state has the effect of lowering the fission barrier by this amount as illustrated in the insert of Fig. 7

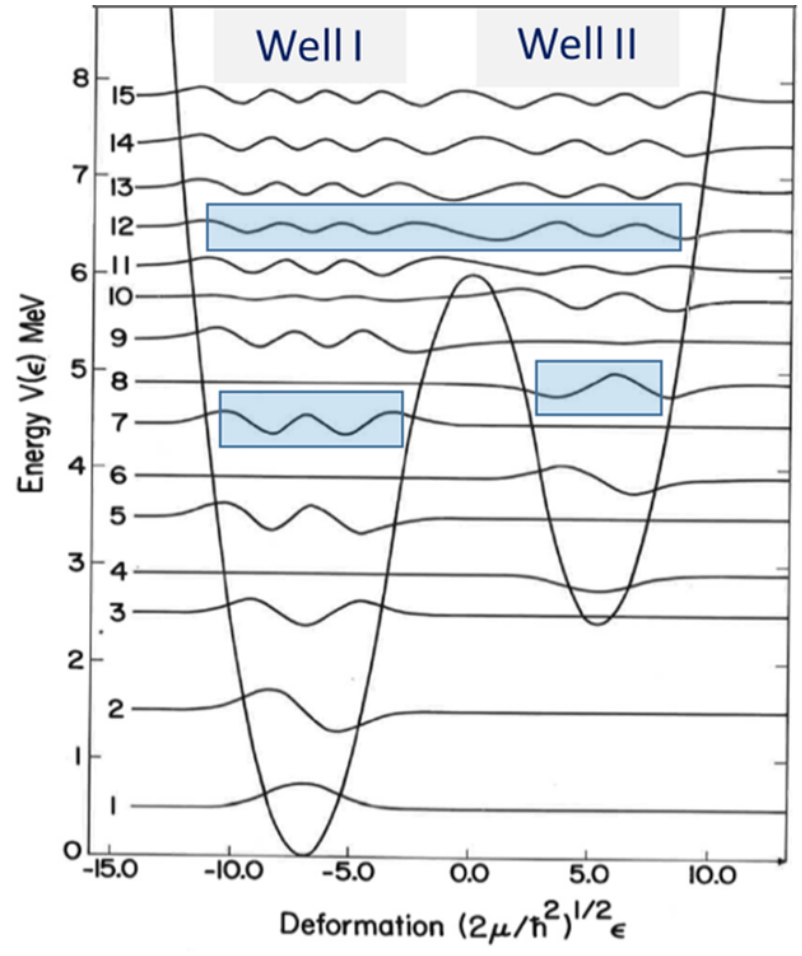

Figure 5. Illustration of $\beta$-vibrational states in a two-well potential composed of three smoothly joined parabolas. Note the fission isomer located in the second well (state \#4) and the mixing of Well I and Well II states occurring near and above the intermediate barrier. [B. B. Back, (1972) unpublished].

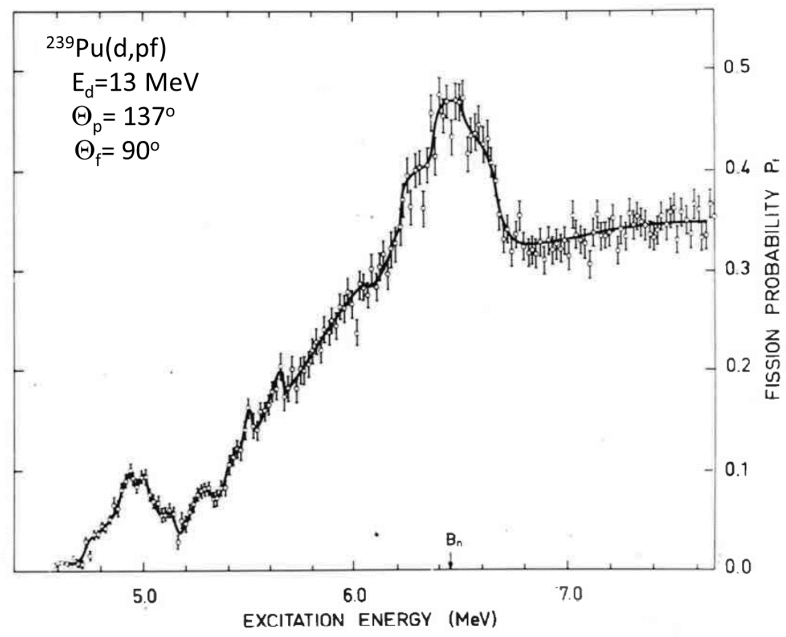

Figure 6. The fission probability for ${ }^{239} \mathrm{Pu}(\mathrm{d}$, pf) is shown as a function of excitation energy. The resonance at $\mathrm{E}_{X} \sim 5.0 \mathrm{MeV}$ corresponds to a $\beta$-vibrational resonance in the second well near the top of the fission barriers [15].

\subsection{Fission barrier parameters}

Analysis of the fission probability function, such as those shown in Figs. 6 and 7 , allow one to derive relevant parameters for the double-humped fission barrier within a statistical model. Assuming that the barrier can be approximated by three smoothly joined parabolas, this leads to 


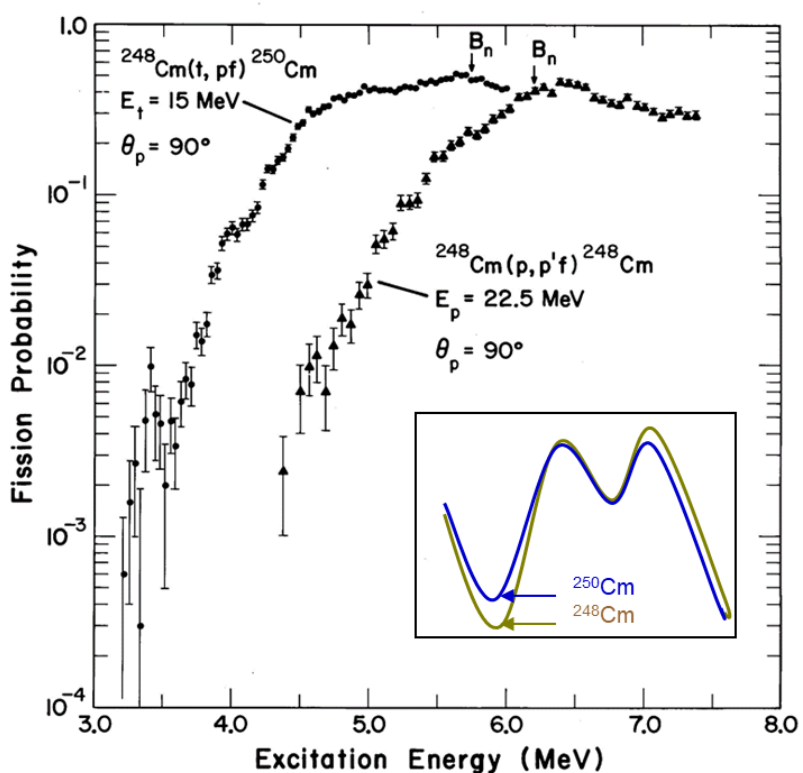

Figure 7. The fission probability functions for ${ }^{248} \mathrm{Cm}$ and ${ }^{250} \mathrm{Cm}$ populated in the ${ }^{248} \mathrm{Cm}$ (p, p'f) and ${ }^{248} \mathrm{Cm}(\mathrm{t}, \mathrm{pf})$ reactions, respectively, show a large $\sim 1 \mathrm{MeV}$ shift caused by the closed $\mathrm{N}=126$ shell lowering the ground state energy of ${ }^{248} \mathrm{Cm}$ relative to ${ }^{250} \mathrm{Cm}$ while the fission barriers are not appreciably affected [19].

six parameters, namely the heights of the inner and outer barrier, $\mathrm{E}_{A}$ and $\mathrm{E}_{B}$, the height of the second well relative to the ground state, $\mathrm{E}_{I I}$, and their curvatures, $\hbar \omega_{A}, \hbar \omega_{B}$, and $\hbar \omega_{I I}$, respectively. Although these parameters are not uniquely determined from fits to the fission probability function alone, some constraints from other observational data and general trends can be applied to derive the main parameters with some level of fidelity. Fig. 8 shows fission barrier parameters obtained from the range of data available for even-even nuclei.

\section{Total kinetic energy - dynamics}

The dynamics of the fission process is an interesting aspect that has received much attention in recent years. One observable that is sensitive to the dynamical properties of the nuclear fluid is the total kinetic energy, TKE, release in fission. This quantity depends strongly on the dissipation taking place during the motion from the saddle point to scission and therefore how much of this potential energy gain is converted to internal heat of the system. An intriguing observation is that the total kinetic energy does not reflect the total energy of the system. For example, one finds that the TKE actually decreases as more excitation energy is imparted into the system as seen in the ${ }^{241} \mathrm{Pu}\left({ }^{3} \mathrm{He}, \alpha \mathrm{f}\right)$ reaction for certain mass splits [21]. This effect is shown in Fig. 9, where one observes that the TKE for the fragment mass bin $\mathrm{M}_{\mathrm{H}}=131-135 \mathrm{u}$, decreases by about $0.8 \mathrm{MeV}$, when the excitation energy is increased by $1 \mathrm{MeV}$. With reference to the right panel of Fig. 9 one notices that the maximum negative slope occurs near

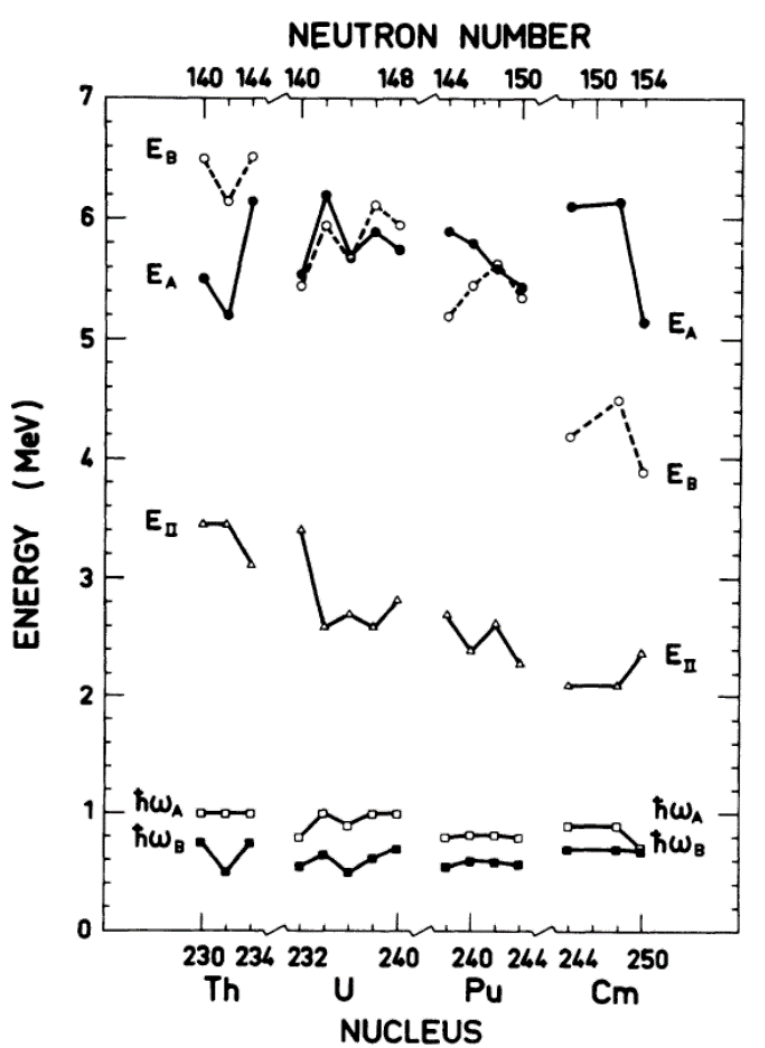

Figure 8. Fission barrier parameters (see text for definition) are shown for fifteen even-even actinide nuclei [20].

$\mathrm{M}=132 \mathrm{u}$, where also the TKE has a maximum. It is natural to assume that both effects are related to the doubly magic ${ }^{132} \mathrm{Sn}$ nucleus. The spherical nature of this nucleus may lead to a compact scission shape, which maximizes the Coulomb repulsion between the fragments. However, increasing the excitation energy reduces the effect of the ${ }^{132} \mathrm{Sn}$ shell leading to a lower TKE. Note that the maximum of the fragment mass distribution occurs for a more asymmetric mass split with the heavy fragment of mass of $\mathrm{M}_{\mathrm{H}} \sim 138 \mathrm{u}$. It is believed that this is caused by a deformed shell for fragments near this mass.

\section{Surrogate reactions}

There is presently much interest in obtaining reliable methods for estimating neutron-induced cross sections for a wide range of materials that cannot be measured directly because of non-availability of the target material. Light charged particle reactions used as surrogates for the neutron capture process appears to have much merit as a way to estimate the neutron capture cross sections with a wide range of applications including nuclear astrophysics, nuclear reactor design and nuclear weapons stewardship. The method relies on the assumption that the neutron capture cross section, $\sigma_{n}$, can reliably be calculated as a function of neutron energy, $E_{n}$, using theoretical models and that the branching ratio for a specific decay channel can be obtained from the charged particle reaction measurement. For the fission channel we thus assume that the following 

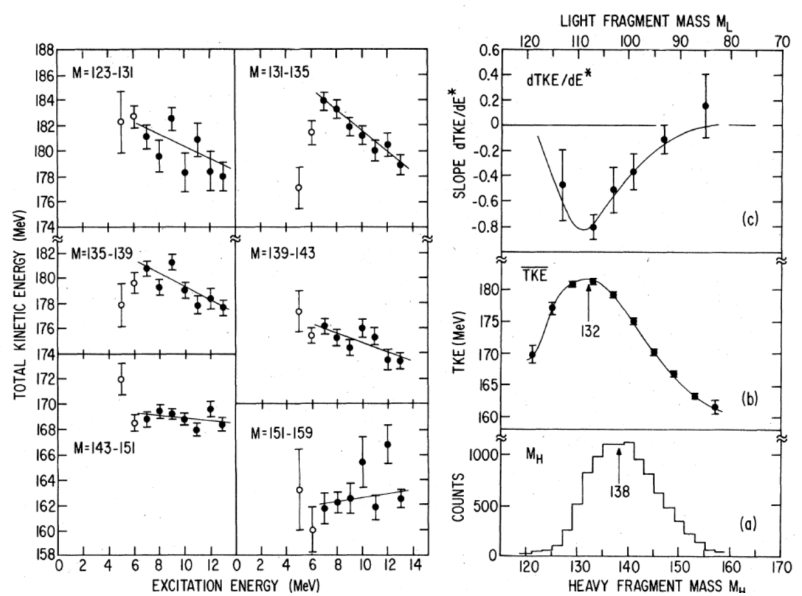

Figure 9. Left panel: The total kinetic energy of fission fragments from the ${ }^{241} \mathrm{Pu}\left({ }^{3} \mathrm{He}, \alpha \mathrm{f}\right)$ reaction is plotted for different heavy fragment mass bins. Solid lines represent best fits to the data over the $\mathrm{E}_{X}=7-13 \mathrm{MeV}$ range. Right panel: The heavy fragment mass distribution (a), the total kinetic energy (b) and the TKE slope with excitation energy (c) are plotted as a function of the ${ }^{240} \mathrm{Pu}$ excitation energy. Figure taken from Ref. [21].

relation is valid

$$
\sigma(n, f)=P_{f}\left(E_{n}+B_{n}\right) \times \sigma_{n}\left(E_{n}\right)
$$

An early application of this method was carried out by Cramer and Britt [22]. It is important to validate the method on cases where actual data on the neutron capture reaction exist. One such case is illustrated in Fig. 9 taken from Ref. [23]. In this work, the fission probability function measured in the ${ }^{234} \mathrm{U}(\mathrm{t}, \mathrm{pf})$ reaction is reasonably well, but not perfectly, reproduced in a statistical model calculation given by the solid curve in the upper panel of Fig. 9. Using this information and correcting for the difference in angular momentum transfer in $(t, p)$ and neutron capture reactions, the authors obtain the estimated $(\mathrm{n}, \mathrm{f})$ cross section shown as solid points in the lower panel of Fig. 9 and compared with the ENDF-B/VI evaluation of measured $(\mathrm{n}, \mathrm{f})$ cross sections (dashed curve). One observes that the $(n, f)$ cross sections obtained by the surrogate reaction method is in good agreement with the evaluated data although there are some deviation in the $\mathrm{E}_{n}=0.1-0.7 \mathrm{MeV}$ range. Currently, much effort is devoted to refine and validate this method [24, 25] as it has a number of important applications as mentioned above.

\section{Opportunities for the future}

Above, I have presented some of the information about nuclear fission processes obtained via light, charged- particle reactions. For various reasons, these types of experiments represent only a cursory look at the issues, partly because the availability of heavy ion beams from a number of facilities drew the attention of many of the practitioners of this field. Consequently, a further detailed study of light ion induced fission was abandoned despite the fact that many new discoveries are surely waiting to be made.

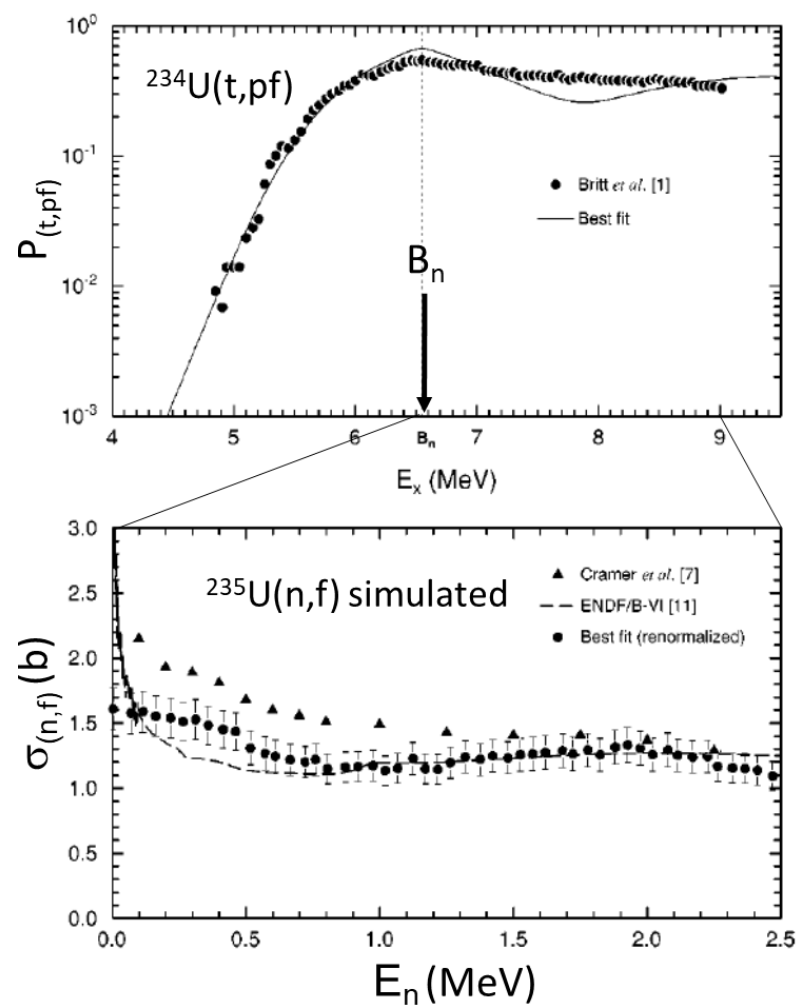

Figure 10. Top panel: The experimental fission probability measured in the ${ }^{234} \mathrm{U}(\mathrm{t}, \mathrm{pf}$ ) reaction (solid points) is shown as a function of excitation energy in ${ }^{236} \mathrm{U}$. The solid line is the best fit to these data using statistical model. Bottom panel: The derived $(\mathrm{n}, \mathrm{f})$ cross section (solid points) are compared with the ENDFB/VI compilation of ${ }^{235} \mathrm{U}$ (n,f) cross sections (dashed line) and the previous surrogate result from Ref. [22] (solid triangles).

Higher precision measurements of the fission probability are for example needed for refining the important approach of using surrogate reactions to predict neutron induced fission cross sections in cases where direct measurements are not possible. In addition, the future availability of heavy radioactive (actinide) beams from e.g. the FRIB facility opens a large area of the nuclear chart for fission measurements using inverse light charged particle reactions as indicated by the red oval in Fig. 10. Radioactive, secondary beams in this range are predicted to have intensities in the range needed for inverse kinematics fission measurements, i.e. $>10^{3} \mathrm{pps}$. Extending the region where reliable fission barrier data are known will place additional constraints on theoretical models and improve the trustworthiness of extrapolations into areas of the nuclear chart, where measurements cannot be made. Of specific interest for astrophysics is the area given by $\mathrm{Z}<82$ and $\mathrm{N}>126$ where the fission recycling in the rapid neutron capture process has been suggested to occur. It is encouraging that instruments that are well suited for such studies are presently available or under construction at facilities where these beams will be available, such as ISS at HIE-ISOLDE and SOLARIS at FRIB. 


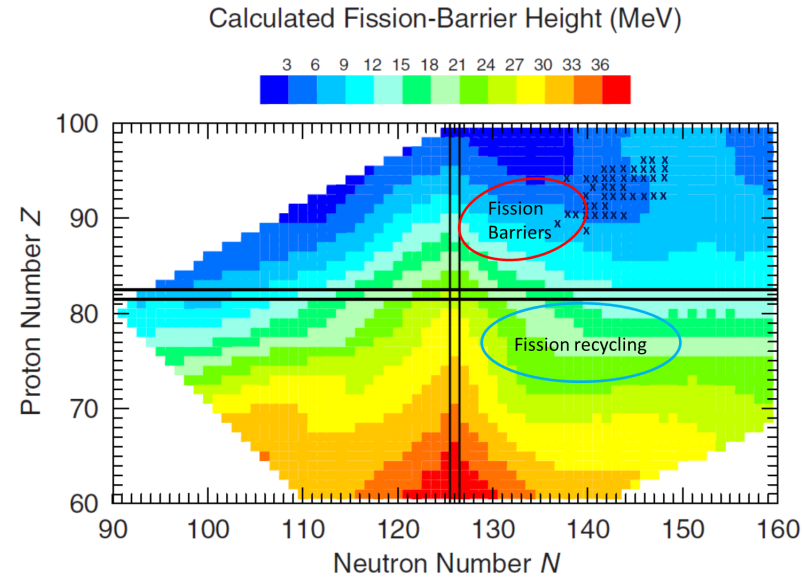

Figure 11. Z-N contour map of predicted fission barrier heights predicted by the macroscopic microscopic liquid drop model by Möller et al. [26]. The crosses indicate the area where fission barriers have been measured using light charged particle beams and actinide targets. The red oval indicates the area where radioactive beams of sufficient intensity to perform inversekinematics fission barrier measurements are expected from the FRIB facility. Unfortunately, predicted intensities are too weak to explore the region proposed for fission recycling (magenta oval). The figure is taken from Ref. [26] and slightly modified.

\section{Summary}

In this talk, I have summarized some of the results concerning the fission process that have been obtained via light charged particle reactions. This method offers excellent control of the excitation energy of the fissioning system spanning the range from far below to well above the fission barrier. Consequently, most of the available information on fission barrier heights and structure has been acquired using this approach. It is also clear that in many cases, these studies were focused on characterizing the multi-peaked structure of the fission barrier, which was a novel aspect of fission research at the time. Subsequently, it has been realized that better and more accurate data are needed, especially at energies above the neutron binding energy, in order to use measured fission probability functions in surrogate reaction analyses to derive $(\mathrm{n}, \mathrm{f})$ cross sections in cases where direct measurements are not feasible. New data that take this aspect into account are clearly needed in order to increase the accuracy of this type of analysis. It may, for example, be important to adjust the angular range for a $(\mathrm{d}, \mathrm{pf})$ reaction such that the transferred angular momentum approximately matches that of the $(n, f)$ reaction with an emphasis on s- and pwaves. In addition, it was pointed out that the availability of radioactive beams in the actinide region allows for studies of fission properties of a large range of nuclei using the inverse kinematics technique. Such beams will become available in the near future at facilities such as FRIB and HIE-ISOLDE. Fortunately, the instrumentation at both of these facilities will include solenoidal spectrometers of the HELIOS type, SOLARIS at FRIB and ISS at HIE-ISOLDE, that circumvent the kinematic compression associated with inverse kinematics. It is thus reasonable to expect that the near future will see a revival of charged particle induced fission studies at these facilities.

\section{Acknowledgement}

This material is based on work supported by the U.S. Department of Energy, Office of Science, Office of Nuclear Physics under contract number DEAC02-06CH11357. This work is dedicated to my $\mathrm{PhD}$ thesis advisors, Sven Bjørnholm and Harold C. Britt.

\section{References}

[1] O. Hahn and F. Strassmann, Naturwissenschaften 27, 11 (1939); L. Meitner and O. R. Frisch, Nature 143, 239 (1939).

[2] N. Bohr and J. A. Wheeler, Phys. Rev. 56, 426 (1939).

[3] W. D. Myers and W. J. Swiatecki, Nucl. Phys. 81, 1 (1966).

[4] S. M. Polikanov et al., Zh. Eksp. Teor. Fiz. 42, 1016 (1962).

[5] G. N. Flerov and S. M. Polikanov, Compt. Rend. Cong. Int. Phys. Nucl. (Paris), 1, 407 (1964).

[6] S. Bjørnholm and V. M. Strutinsky, Nucl. Phys. 136, 1 (1969).

[7] V. M. Strutinsky, Ark. Fys. 36, 629 (1967); Nucl. Phys. A 95, 420 (1967).

[8] J. A. Northrop, R. H. Stokes, and K. Boyer, Phys. Rev. 115, 1277 (1959).

[9] W. Kolar and K. H. Böckhoff, J. Nucl. Energy 22, 299 (1968).

[10] E. Migneco and J. P. Theobald, Nucl. Phys. A 112, 603 (1968).

[11] S. Bjørnholm and J. E. Lynn, Rev. Mod. Phys. 52, 725 (1980).

[12] P. J. Twin et al., Phys. Rev. Lett. 57, 811 (1986).

[13] E. F. Moore et al., Phys. Rev. Lett. 63, 360 (1989).

[14] H. J. Specht et al., Phys. Lett B 41, 43 (1972).

[15] B. B. Back et al.,in Proc. of the Sec. Int. Symp.on the Phys. and Chem. of Fission, Vienna, 1969, p. 351, Vienna, 1969.

[16] P. Glässel, H. Rösler, and H. J. Specht, Nucl. Phys. A 256, 220 (1976).

[17] A. Krasznahorkay et al., Phys. Rev. Lett. 80, 2073 (1998).

[18] M. Csatlós et al., Phys. Lett. B 615, 175 (2005).

[19] B. B. Back et al., Phys. Lett. B 46, 183 (1973).

[20] B. B. Back et al., Phys. Rev. C 9, 1924 (1974).

[21] B. B. Back, J. Lebowitz, K. Wolf, Phys. Rev. C 20, 1819 (1979).

[22] J.D. Cramer and H. C. Britt, Nucl. Sci. Eng. 41, 177 (1970).

[23] W. Younes and H. C. Britt , Phys. Rev. C 67, 024610 (2003).

[24] J. E. Escher and F. S. Dietrich, Phys. Rev. C 74, 054601 (2006).

[25] G. Kessedjian et al., Phys. Lett. B 692, 297 (2010).

[26] P. Möller et al., Phys. Rev. C 91, 024310 (2015). 